\title{
AN ELEMENTARY EXAMPLE IN $p$-ADIC DIOPHANTINE APPROXIMATION ${ }^{1}$
}

\author{
RICHARD T. BUMBY
}

1. Introduction. It is the purpose of this note to call attention to the possibility of constructing $p$-adic examples which are related to unsolved problems in classical diophantine approximation. The significance of such examples rests on the principle that the theorems of classical diophantine approximation can be modified to obtain $p$-adic analogs. It should even be possible to prove that certain classical methods "automatically" extend to the $p$-adic case. We shall not concern ourselves here with the exact nature of the analogy between classical diophantine approximation and the $p$-adic generalizations. We shall, however, assume that some precise form of this principle can be found in a particular case where we have observed an analogy.

2. Diophantine approximation. We shall understand the term "diophantine approximation" to refer to problems of the following type. A function $f(x, y)$ will be given whose domain is the set of pairs of integers and whose range is the set of non-negative real numbers. We shall consider inequalities of the form $f(x, y)<c$ for all $c>0$. The question asked will be: For which $c$ are there infinitely many solutions to $f(x, y)<c$ in relatively prime integers $x, y$ ? The realm of classical diophantine approximation concerns itself with functions $f$ which can be thought of as functions of real variables; $p$-adic diophantine approximation allows functions which involve computations in the various $p$-adic completions of the rationals, and computation of the $p$-adic valuation on elements of that completion. ${ }^{2}$ form

In this note, we shall consider problems based on functions of the

$$
g(\max (|x|,|y|)) \cdot|x| \cdot|y| \cdot|x|_{2} \cdot|y|_{3} \cdot|x-y|_{5}
$$

where ||$_{p}$ denotes the $p$-adic valuation. The principle we are discussing indicates that these problems should be related to problems in classical diophantine approximations involving

$$
g(\max (|x|,|y|)) \cdot|y| \cdot|\alpha y-x|
$$

\footnotetext{
Received by the editors October 20, 1962.

${ }^{1}$ This example is a by-product of the author's Ph.D. thesis [1].

2 The definitions of $p$-adic completions and valuations may be found in $[5,876]$ or $[3$, p. 5].
} 
for algebraic $\alpha$. The next section should give an indication of the relationship between classical and p-adic problems.

3. $g(z)=z^{\epsilon}, \epsilon>0$. The case in which $g(z)=z^{\epsilon}, \epsilon>0$, is covered by Roth's theorem. In the classical case, Roth's original theorem demonstrates that

$$
(\max (|x|,|y|)) \cdot|y| \cdot|\alpha y-x|<c
$$

will have only finitely many solutions in relatively prime integers for all $c>0$. The $p$-adic analogs of Roth's theorem (which were proved by others shortly after Roth's result became known) indicate that the same conclusions hold for the $p$-adic generalizations of (3). (See [3].) In particular, we have that

$$
(\max (|x|,|y|))^{e} \cdot|x| \cdot|y| \cdot|x|_{2} \cdot|y|_{3} \cdot|x-y|_{5}<c
$$

will have only finitely many solutions in relatively prime integers for all values of $c>0$.

4. $g(z)=1$. If, instead, we take $g(z)=1$ in the classical case, so that we are concerned with inequalities of the form

$$
|y| \cdot|\alpha y-x|<c
$$

then the continued fraction of $\alpha$ allows us to construct infinitely many solutions to (5) with $c=1$ if $\alpha$ is irrational. If $\alpha$ were rational, it is easy to see that (5) must have only finitely many solutions in relatively prime integers for each $c$. If $\alpha$ is of degree 2 over the rationals, then $\alpha$ will have a periodic continued fraction, a fact which allows us to prove that there will be a value of $c$ for which (5) has only finitely many solutions. If the degree of $\alpha$ is greater than 2 , we do not know if there is any value of $c$ such that (5) has only finitely many solutions. (See $[3$, p. vi].)

The particular $p$-adic analog which we have described is easier to deal with than its classical counterpart. We can easily construct a sequence of pairs $\left(x_{n}, y_{n}\right)$ such that

$$
\left|x_{n}\right| \cdot\left|y_{n}\right| \cdot\left|x_{n}\right|_{2} \cdot\left|y_{n}\right|_{3} \cdot\left|x_{n}-y_{n}\right|_{s}=5^{-n} \text {. }
$$

Theorem 1. A sequence of pairs $\left(x_{n}, y_{n}\right)$ satisfying (6) is formed by setting $x_{n}=2^{2 \cdot 5^{n-1}}$ and $y_{n}=-1$.

Proof. The choices we have made for $x_{n}$ and $y_{n}$ demonstrate that $\left|x_{n}\right| \cdot\left|x_{n}\right|_{2}=1$ and $\left|y_{n}\right| \cdot\left|y_{n}\right|_{3}=1$. Hence, we must show that $\left|x_{n}-y_{n}\right|_{5}=5^{-n}$. Translating this into the language of elementary number theory, we find that we must show that 


$$
2^{2 \cdot 5^{n-1}} \equiv-1\left(\bmod 5^{n}\right)
$$

and

$$
2^{2 \cdot 5^{n-1}} \not \equiv-1\left(\bmod 5^{n+1}\right) .
$$

These results in turn follow from the fact that 2 is a primitive root modulo $5^{n}$ for all $n$. This may be verified easily by using the following result from elementary number theory.

LEMMA. If $r$ is relatively prime to the odd prime $p$, and if $r$ is a primitive root modulo $p^{2}$, then $r$ is a primitive root modulo $p^{n}$ for all $n$.

REMARK. This lemma is a consequence of the binomial theorem; a proof may be found in [2, Vol. 1, Theorem 4-6, p. 52] or [4, p. 108].

Although we know of no algebraic number $\alpha$ for which (5) has infinitely many solutions for all $c$, we have constructed an analogous $p$-adic problem for which this is the case. This demonstrates that any proof that all algebraic numbers have continued fractions with bounded partial quotients would have to be such that it not admit of extension to the obvious $p$-adic analog. Considering the methods of diophantine approximation, this seems to be fair evidence for assuming that this conjecture is false. While this does not bring us much nearer to final resolution of this problem, it does provide some evidence that algebraic numbers might exist for which (5) has infinitely many solutions for all $c>0$.

5. $g(z)=\log z$. The sequence of pairs $\left(x_{n}, y_{n}\right)$ which we constructed above can be used to prove a stronger result than that for which it was introduced.

THEOREM 2. There is a constant $c>0$ such that

$$
\log (\max (|x|,|y|)) \cdot|x| \cdot|y| \cdot|x|_{2} \cdot|y|_{3} \cdot|x-y|_{5}<c
$$

has infinitely many solutions in relatively prime integers.

Proof. Direct computation with the sequence $\left(x_{n}, y_{n}\right)$ constructed in Theorem 1 shows that this holds for $c>(2 / 5) \log 2$.

Remark. A smaller value of $c$ may be obtained by the following argument.

First observe that 2 and 3 are both primitive roots of $5^{n}$ for all $n$. Also observe that, if $x= \pm 2^{a}$ and $y= \pm 3^{b}$, then (7) reduces to

$$
\log (\max (|x|,|y|)) \cdot|x-y|_{5}<c .
$$

Now let 


$$
A_{n}=\left\{ \pm 2^{a}: 0 \leqq a \leqq 2 \cdot 5^{n-1} \frac{\log 3}{\log 6}\right\}
$$

and

$$
B_{n}=\left\{ \pm 3^{b}: 0<b \leqq 2 \cdot 5^{n-1} \frac{\log 2}{\log 6}+1\right\} .
$$

It is clear that the elements of $A_{n}$ are distinct modulo $5^{n}$, and also the elements of $B_{n}$ are distinct modulo $5^{n}$. Since $A_{n} \cup B_{n}$ contains a number of elements greater than $4 \cdot 5^{n-1}$, all of which are relatively prime to 5 , this set can not consist of elements distinct modulo $5^{n}$. Select $x_{n} \in A_{n}, y_{n} \in B_{n}$ such that

$$
x_{n} \equiv y_{n}\left(\bmod 5^{n}\right)
$$

If $c>(2 / 5)(\log 2)(\log 3) / \log 6$ is given, then we will have

$$
\log \left(\max \left(\left|x_{n}\right|,\left|y_{n}\right|\right)\right) \cdot\left|x_{n}-y_{n}\right|_{6}<c
$$

for all $n>n_{0}(c)$.

\section{REFERENCES}

1. R. T. Bumby, On the foundations of diophantine approximation, Ph.D. thesis, Princeton Univ., Princeton, N. J., 1962.

2. W. J. LeVeque, Topics in number theory, 2 vols., Addison-Wesley, Reading, Mass., 1956.

3. K. Mahler, Lectures on diophantine approximations. Part 1. g-adic numbers and Roth's theorem, Cushing-Malloy, Ann Arbor, Michigan, 1961.

4. T. Nagell, Introduction to number theory, Wiley, New York, 1951.

5. B. L. van der Waerden, Algebra. I, Springer, Berlin, 1955. (Earlier editions, known as Moderne Algebra, essentially the same.)

Rutgers, The State University 